This is an Open Access article distributed under the terms of the Creative Commons Attribution-Noncommercial License, which permits unrestricted use, distribution, and reproduction in any noncommercial medium, provided the original work is properly cited.

\title{
DEVELOPMENT OF A LARGE SCALE STRESSED GE:GA DETECTOR ARRAY FOR SAFARI
}

\author{
W. Raab, A. Poglitsch, R. Höhnle, and L. Barl \\ MPE-Garching, Garching, Germany
}

\begin{abstract}
We report on the development of a large format stressed gallium doped germanium (Ge:Ge) array for the SAFARI instrument. Building on flight proven PACS heritage, the goal of our development is a 32 pixel stressed Ge:Ga module enabling a $32 \times 32$ pixel photoconductor array for the wavelength range between 110 and $210 \mu \mathrm{m}$. The unprecedented size of this array would allow the use of almost all of the $3.8 \times 3.8$ arcmin field of view provided for SAFARI in the SPICA focal plane. We are currently in the process of manufacturing the individual components of the 32 pixel demonstration module. This prototype module will feature three selectable read out architectures enabling the evaluation and optimisation of the detector performance as well as a two stage multiplexer to distribute the dissipative heat load on the temperature levels provided by the satellite. Thermal modeling has shown that the heat loads are in compliance with the thermal budgets of the SPICA cryogenic system. The ultimate development goal with optimised read out circuits is an NEP of $1 \times 10^{-18} \mathrm{~W} / \mathrm{Hz}^{1 / 2}$, which would present a factor of 8 improvement in the noise performance compared to the PACS stressed Ge:Ga array.
\end{abstract}

Key words: Missions: SPICA - Detectors: Photoconductors - stressed Ge:Ga

\section{INTRODUCTION}

Semiconductor based detectors have been used to detect infrared radiation since the early days of space astronomy, and are now literally the workhorse of infrared space missions. Whilst their leading position in space applications is starting to be challenged by the promise of largeformat, high-performance bolometer arrays, semiconductor devices currently still are the most sensitive detector type in operation at infrared wavelengths. Photoconductors offer an enormous flight heritage. Instruments using these detectors include among many others: LWS and ISOPhot on ISO (various detectors including: $3 \times 3$ pixels Ge:Ga, $2 \times 2$ pixels stressed Ge:Ga) (Clegg et al., 1996; de Graauw et al., 1996), PACS on Herschel $(16 \times 25$ pixels Ge:Ge, $16 \times 25$ pixels stressed Ge:Ge) (Poglitsch et al., 2008 ), and FIS on AKARI (up to $20 \times 3$ pixels monolithic Ge:Ga, up to $15 \times 3$ pixels stressed Ge:Ga) (Kawada et al., 2007). Based on the available photoconductor materials to date the photoconductor working group proposes to cover the wavelength range between $\tilde{4} 5$ and $210 \mu \mathrm{m}$ in two separate bands, using a monolithic Ge:Ga array (Fujiwara et al., 2003) for the "short" wavelength band between 45 and $110 \mu \mathrm{m}$ and a stressed Ge:Ga array for the "long" wavelength band between 110 and $210 \mu \mathrm{m}$. In addition to these two bands, the new development of CSIP (Charge Sensitive Infrared Phototransistor) (Ueda et al., 2008) arrays potentially offers an extension of the SAFARI bands towards shorter wavelengths. This article describes the design of a 32 pixel stressed Ge:Ga module. The design of the proposed monolithic arrays as well as the development status of the CSIP devices can be found elsewhere in these proceedings.

\section{Key Characteristics}

The proposed stressed photoconductor module described here is directly based on the design of the very successful 16 pixel stressed Ge:Ga module of PACS (Kraft et al., 2001). PACS uses a set of 25 such modules to form the $16 \times 25$ pixel long-wavelength array. For SAFARI we propose to built a 32 pixels stressed Ge:Ga module being the building block of a $32 \times 32$ pixels detector array. The module will be stressed to a cut-off wavelength of $210 \mu \mathrm{m}$, resulting in an operating wavelength range between 110 and $210 \mu \mathrm{m}$. Assuming Nyquist sampling at the center of the long waveband $(160 \mu \mathrm{m})$, the planned array would allow an instantaneous field of view $3 \times 3$ arcmin. The physical dimensions of the individual semiconductor pixels is $1 \times 1 \times 1.5 \mathrm{~mm}$. The arrangement of the individual pixels in the module (see section 3 ) is leading to a pixel size of $3.6 \times 3.6 \mathrm{~mm}$ in the focal plane. The total mechanical envelope of the stressed array is $179 \times 128 \times 85 \mathrm{~mm}$. Each module will have a mass of $110 \mathrm{~g}$, resulting in a total mass of the entire array of $\tilde{3} .5 \mathrm{~kg}$. As detector sensitivity is the main development driver, the development of ultra low-noise, ultra low-power cryogenic read-out electronics is one of the most important goals of the photoconductor program.

\section{Module Design}

Figure 1 shows the proposed design of the 32 pixels stressed Ge:Ga module for SAFARI. The module consists of the 
actual module body itself, including the stressing springs, the linear fore-optics (feed horns) and two substrates carrying the read-out electronics (CRE substrate) and the multiplexer electronics (MUX substrate). The figure shows how individual Ge:Ga pixels are stacked on top of each other to form one linear detector module. Mechanical stress onto the stack is applied by two spring-loaded stressing screws on either side of the stack. The pixel stack is mounted inside a highly reflective, gold coated integrating cavity and is covered by a fore optics of feed horns forming optical concentrators. The arrangement of pixels, stressing pistons, insulating shims and spherical segments used to compensate uneven and non-parallel pixel surfaces is illustrated in the exploded view. This arrangement of the individual pixels is identical to the one used in the PACS arrays. A solid "stack divider" separates the stack itself mechanically into two sections of 16 pixels each. All mechanical problems resulting from the increased numbers of pixels thereby are effectively reduced to levels successfully demonstrated by the 16 pixel PACS modules.

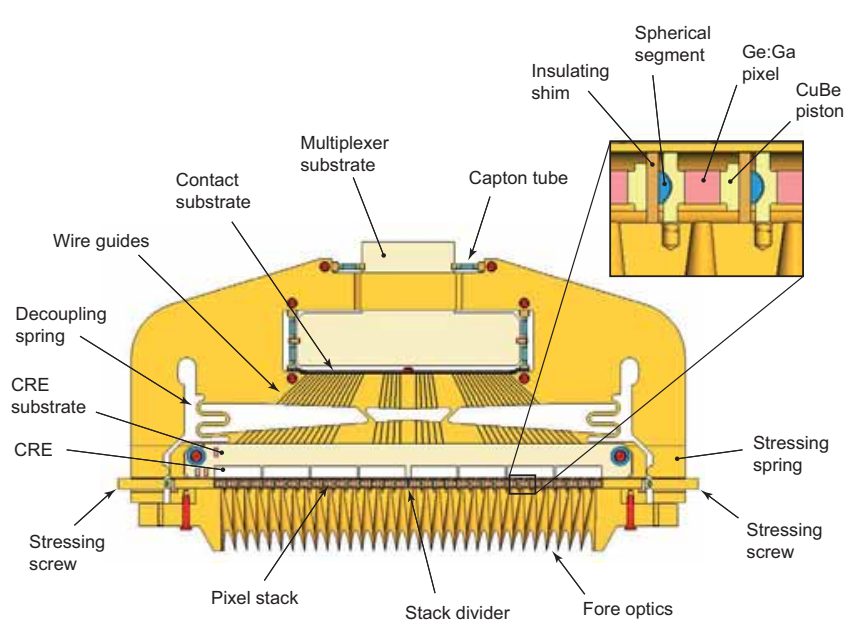

Figure 1. Design of the 32 pixel stressed Ge:Ga module proposed for SAFARI. The small insert shows the assembly of the individual components of the stack.

The individual pixels are mounted between two $\mathrm{CuBe}$ pistons providing electrical contact to ground on one end and to the bias voltage on the other end. The ground pads are in direct contact to the module housing. On the bias side, signal wires are mounted on the $\mathrm{CuBe}$ pads which then connect to the input channels of the CRE via insulated micro feed throughs. This design enables the use of very short signal wires and therefore ensures minimal stray input capacities. By this design optimisation alone, an improvement of the noise performance by a factor of 2 is expected compared to the PACS array. In total, eight cryogenic read-out chips (CREs) carrying four input channels each are mounted on a common CRE substrate in close proximity to the pixel stack.
The integrated signals and the wires carrying the supply voltages and digital signals required for the operation of the CREs are routed through wire guides to a contact substrate and from there to the multiplexer substrate. The multiplexer substrate carries a two stage multiplexer electronics. To distribute the heat load induced by the dissipation of the cryogenic electronics, the multiplexers are operated at the $4.5 \mathrm{~K}$ level (as opposed to the CRE, which is operated at $1.7 \mathrm{~K}$ ). The multiplexer substrate is thermally insulated from the actual detector module by a set of thin walled capton tubes. The cryogenic read out architecture and the multiplexing scheme are discussed in detail in the next section.

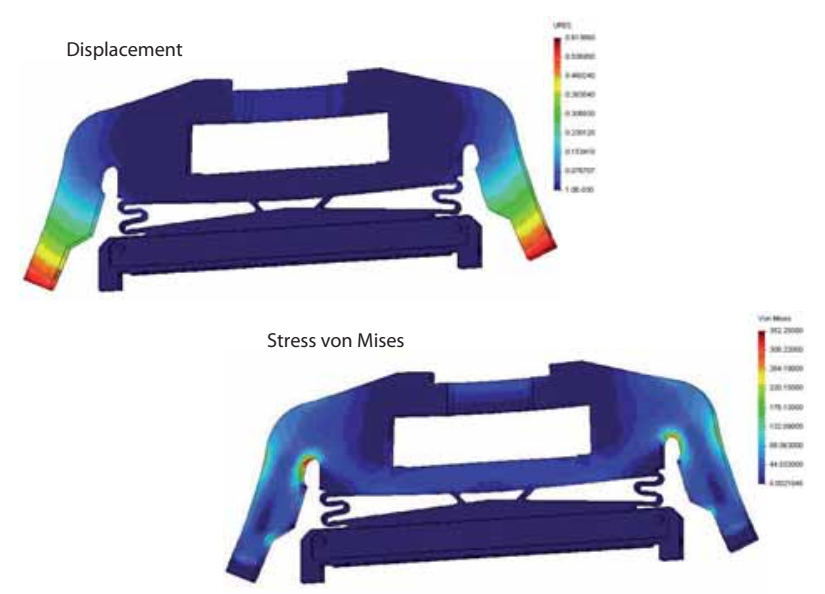

Figure 2. Finite element analysis of the stressed Ge:Ge module. The top left figure shows the displacement (in millimetres) and the bottom right figure the distribution of von Mises stress (in $M P a)$. Note: the actual displacement shown in the figures is exaggerated by a factor of 10 .

It is extremely important to ensure that the actual pixel stack stays aligned along a straight line at all times. Any bending or deformation of the stack would lead to non-uniform stress in the individual pixels and consequently to an uneven distribution of the wavelength response or even breaking of pixels and in consequence the collapse of the entire stack in the most extreme case. The module therefore is designed to ensure the mechanical decoupling of the part of the module containing the pixel stack from the actual body of the module with the stressing spring. To verify the design, extensive finite element analysis has been carried out. The main findings in terms of distribution of the stress (von Mises) and the displacement are shown in Figure 2. The figures show that both stress and displacement are negligible in the vicinity of the pixel stack.

\section{Read-out Electronics And Multiplexing}

The most important read-out architectures for the photoconductors in the SAFARI project are the Direct Injection 
(DI), the Buffered Direct Injection (BDI) and Capacitive Transimpedance Amplifier (CTIA) cells (Figure 3). Each of these topologies offers a number of advantages and disadvantages for the proposed arrays. Most importantly, the DI architecture features very low power consumption, very good noise performance and low complexity, but very little bias control leading to large variations of bias voltage across the detector pixels. The CTIA architecture on the other hand offers excellent bias stability at the cost of high power consumption, high complexity and only moderate noise performance. The BDI as the third architecture under investigation in many ways is a compromise between the DI and the CTIA architecture which also reflects in its properties: better bias control than the DI but lower power consumption and better noise performance than the CTIA. As photoconductors exhibit a strong bias dependence in their response, very good bias control is regarded as essential for the operation of these detectors. The CTIA architecture therefore is the preferred technology for the photoconductor arrays.

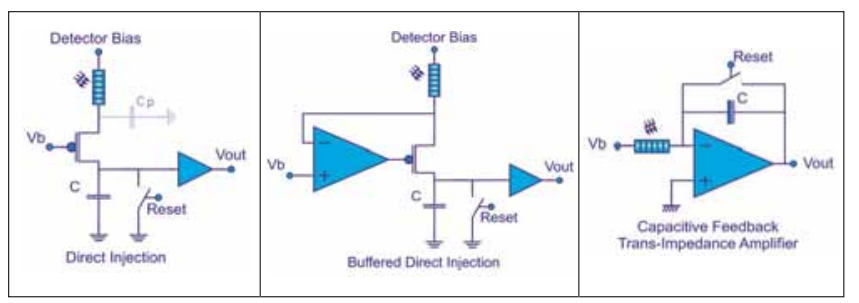

Figure 3. The three read-out topologies under study for SAFARI photoconductors.

All of the aforementioned read-out architectures (CTIA, DI and BDI) are currently under investigation for the stressed array. For an effective evaluation of the properties of each of these topologies, a four channel read-out prototype chip was developed by IMEC in Leuven/Belgium. Each of the four input channels contains all three read-out topologies in parallel. The desired read-out is selected by wire bonding the detector pixel to the respective input. Eight of these cryogenic read-out chips are required to read-out all 32 pixels of the module. As mentioned before, all CRE chips are mounted on a common CRE substrate in close proximity to the pixel stack (see Figure 1).

Multiplexing of the 32 signals is performed by a separate two-stage multiplexer: eight 4:1 multiplexers are used in the first stage and one 8:1 multiplexer in the second stage. All 32 signal lines from the individual pixels are therefore multiplexed into a single output line. The multiplexed signal is carried via a ribbon cable to a buffer and line driver stage at $4.5 \mathrm{~K}$. The ribbon cable also carries all supply voltages as well as logic/digital signals to drive the multiplexers and the CREs. The signal is further routed via a series of buffers/line drivers on the available temperature stages to the detector control unit (DCU) of the

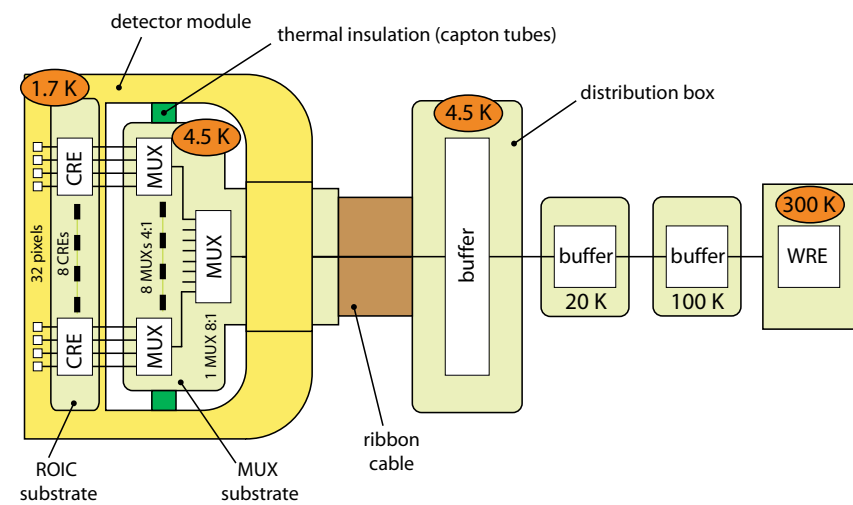

Figure 4. Electrical/mechanic layout of the stressed Ge:Ga module. Lines for supply voltages and logic/control signals are not drawn.

warm electronics. The electrical layout of the module is illustrated in Figure 4.

\section{Thermal Design}

The design of the stressed photoconductor module is to a large extent driven by the necessity of distributing the dissipative heat loads on the available temperature levels and at the same time minimising the parasitic heat load by the cryo-harness cables and support structures. The thermal block diagram shown in Figure 5 summarises the calculated heat loads on the temperature levels provided by the satellite.

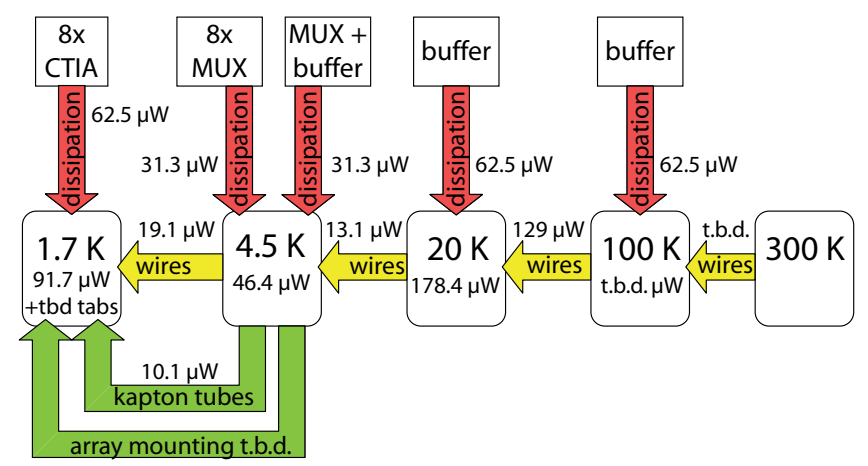

Figure 5. Thermal loads on the temperature levels provided by the SPICA satellite. Red arrows show dissipative heat loads (i.e. electronics), yellow arrows parasitic loads by the cryoharness and green arrows parasitic loads by structural components. The numbers quoted are calculated per module and contain a margin of $50 \%$ for parasitic loads and a margin of $25 \%$ for dissipative loads.

For all structural parts only heat transfer by heat conduction was assumed. This leads to conservative results for the heat load as in reality the heat load is further reduced by the additional (but hard to quantify) thermal resistance at the contact areas. CTIA topology was as- 
sumed for the cryogenic read-out electronics. This again presents the worst case as CTIA circuits by far have the highest power dissipation of all architectures under study. In addition, the calculation of the heat loads includes a margin of $50 \%$ for the cryo-harness and structural components and $25 \%$ for the heat dissipation of the electronics.

\section{Current Status}

As of Summer 2009, the mechanical design of the SAFARI prototype modules including extensive finite element analysis is completed. All components of the pixel stack and the light cones are manufactured and gold coated (where applicable). The only component still missing at the moment is the actual module body itself, but delivery is expected in Autumn 2009. The four channel/three topology CRE prototype circuits, as well as the $4: 1$ and the 8:1 multiplexers were successfully manufactured and delivered (Figure 6). Bread-board testing of the read-out chain at room temperature and the design of the warm read-out electronics has started.
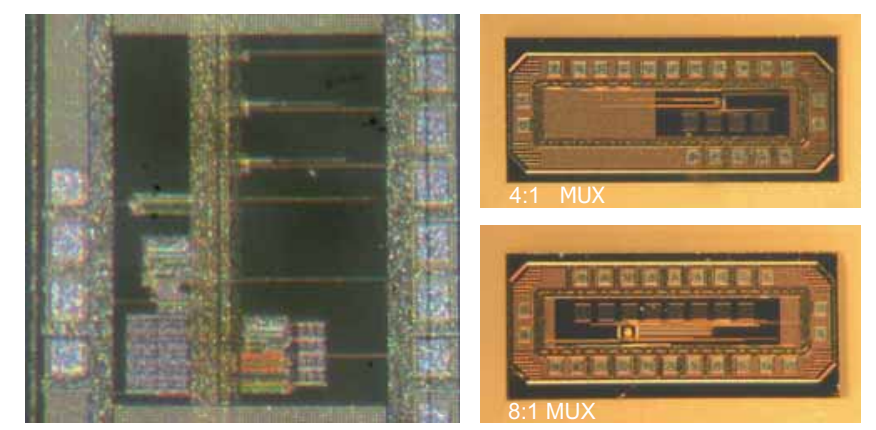

Figure 6. Micro-photography of one of the channels of the CRE and the 4:1 and 8:1 multiplexers designed for the stressed photoconductors by IMEC.

A liquid helium cryostat originally designed for the ISO-SWS instrument and adapted for PACS detector tests is currently modified to accommodate the larger 32 pixel SAFARI array. The cryogenic test setup will include a black body source and a number of mirrors, but no attenuation (or other) filters. This way some well known problems such as the uncertainty of cold transmission curves and filter temperatures as well as multiple reflections between filter stacks can be avoided, leading to reliable flux calculations.

\section{Estimated Performance}

The performance estimates available to date are based on measurements of the PACS stressed Ge:Ga arrays. The cut-off wavelength of the prototype module is expected to be around $\tilde{2} 10 \mu \mathrm{m}$ with a peak responsivity of $\tilde{4} 5 \mathrm{~A} / \mathrm{W}$ at $180 \mu \mathrm{m}$. The best value for NEP derived for the PACS array was $8 \times 10^{-18} \mathrm{~W} / \mathrm{Hz}^{1 / 2}$, measured at the typical PACS background $\left(2.4 \times 10^{-15} \mathrm{~W}\right)$. The NEP of the PACS array is therefore almost a factor of 10 higher than the required $8.6 \times 10^{-19} \mathrm{~W} / \mathrm{Hz}^{1 / 2}$ derived for near background limited performance in the proposed FTS spectrometer of SAFARI. From careful analysis of the PACS measurements, we know that the dominant noise source is the cryogenic read-out electronics. A significant improvement in the detector noise performance is therefore expected with optimised CREs. Although the sensitivity required for BLIP performance can not be reached realistically with stressed photoconductors, the ultimate development goal for the stressed photoconductors is an NEP of $1 \times 10^{-18} \mathrm{~W} / \mathrm{Hz}^{1 / 2}$.

\section{Summary}

The photoconductor development group has investigated the feasibility of a large format stressed Ge:Ge array for the SAFARI. We are confident that a 32 pixel linear stressed module enabling a $32 \times 32$ pixel array is within the scope of this study, and we will provide a fully scale prototype module to enter the detector down selection process. The prototype module will feature three selectable read out architectures enabling the evaluation and optimisation of the detector performance as well as a two stage multiplexer to distribute the dissipative heat load on the temperature levels provided by the satellite. Aware of the fact that stressed photoconductors will most likely not be able to reach the required sensitivity for BLIP performance on SAFARI, the ultimate development goal for the stressed photoconductors still is an NEP of $10^{-18} \mathrm{~W} / \mathrm{Hz}^{1 / 2}$. We are confident, that the maturity of the devices makes them a strong competitor in the detector down selection process.

\section{REFERENCES}

Clegg, P.E., Ade, P.A.R., Armand, C., et al. 1996, A\&A 315, L38

de Graauw, T., Haser, L.N., Beintema, D.A., et al. 1996, A\&A 315, L49

Fujiwara, M., Hirao, T., Kawada, M., et al. 2003, Ap. Opt. 42,2166

Kawada, M., Baba, H., Barthel, P.D., et al. 2007, PASJ $59, \mathrm{~S} 389$

Kraft, S., Merken, P., Creten, Y., et al. 2001, Proc. SPIE 4540, 374

Poglitsch, A., Waelkens, C., Bauer, O.H., et al. 2008, Proc. SPIE 7010, 701005

Ueda, T., An, Z., Hirakawa, K., \& Komiyama, S. 2008, J. Appl. Phys. 103, 093109 